\title{
Improving the Response Performance of Anti-Surge Control Valve for Gas Compressors
}

\author{
Haruna A. Ogweda and Joseph E. Okhaifoh
}

\begin{abstract}
Anti-surge control valves are used for the protection of gas compressors by opening to relief sudden pressures (surge) which could damage the compressor internals. It is of great importance that the response of the anti-surge valves to surge detection is swift and within a few seconds since a little delay could cause catastrophic damages to the compressor it is meant to protect. In this paper, a design modification was done on an existing anti-surge valve to improve its response time. The modified system was designed and implemented, installed, and commissioned on a mixed refrigerant compressor. The modified system was tested, and the response performance was recorded. Results obtained showed average percentage improvement of $86 \%$ to that of the initial system. Also, the response performance of the modified system was less than 3 seconds as compared to the initial set-up which averages 9 seconds. This is indeed a great improvement in comparison to the initial system.
\end{abstract}

Keywords - Anti-surge control valve, Compressor, Actuator, Volume booster, Surge.

\section{INTRODUCTION}

In gas industries, compressors are critical in their operations, so great precautions are usually taken to protect them against damage or destruction due to their delicateness and expensiveness. In normal operation these compressors take processes from the suction vessels, carry out their compression and push same out into the receiver vessels from where the processes are transferred to other part of the plant through a flow discharge valve [1].

The biggest risk they face while in operation is known as the "surge phenomena" [1]. Compressor Surge is the phenomena whereby there is a reverse flow of process through a centrifugal or axial compressor. It can occur when there is increased pressure in the receiver vessel due to decreased flowrate of process into the compressor and hence a decrease in the kinetic energy in the compressor. This invariably leads to a decrease in the pressure in the compressor as compared to that in the receiver vessel leading to a reverse direction of flow across the compressor [2].

This situation is undesirable because when gas flows backward, it pushes the rotating assembly backwards, thereby slamming the rotating shaft into the thrust bearing. In other words, the rotating assembly collides with the static assembly, thereby putting a lot of strain on it and damaging major compressor internals like seals, bearings, blades etc. This obviously leads to down time, loss of production and

Submitted on October 03, 2021.

Published on November 25, 2021.

Haruna A. Ogweda, Federal University of Petroleum Resources Effurun, Nigeria.

(e-mail: ogwedas2000@yahoo.com) very high financial implications for replacement of damaged part.

Compressor surge can be prevented by installing protection system known as a "spill-back valve" (Anti-surge Valve) [2]. These valves have set point above the minimum flow rate (surge point). Once a back flow is detected the antisurge valve opens to allow excess flow back into the suction drum.

The anti-surge valves installed on the gas compressors offer this kind of protection and control through:

a) Fast response - to meet the Multivariable Indicate controller (UIC) demand/expectation.

b) High Reliability - always available upon demand.

The fact that the Anti-Surge Control Valve (ASCV) system protects critical equipment as the compressor from surge makes it a critical equipment whose importance cannot be overemphasized. Over the years, there has been many train trips with anti-surge valves failure being the direct root cause or a contributory factor. This has been evidently identified in the operation of the anti-surge valves in the Nigeria Liquified Natural Gas Train 1 Mixed Refrigerant Compressor. One of the common failures observed with ASCV on site is the sluggish response with stroke time of up to 12 seconds against desired less than 3 seconds.

\section{RELATED WORKS}

When the topical issue of anti-surge control for compressors using anti-surge valves was studied, it was realized that some research had already been done, although without say about the type of actuation these valves employed. Therefore, a summary of the recent studies is included in this work.

[3] designed and developed an anti-surge control system for centrifugal compressors. In the design, they considered a piping system near the compressor for minimizing the volume in the compressor discharge, inlet of the anti-surge valve, non-return check valve and compressor discharge flange as damage to a compressor in a compressor surge is dependent on the volume of gas in the compressor discharge. They introduced a surge detector algorithm in their system for triggering an alarm and shutting down the compressor when other methods of preventing surge failed. The developed anti surge control system operates by measuring and expressing the distance between the current position of operating point and Surge limit line of the compressor by varying the position

Joseph E. Okhaifoh, Federal University of Petroleum Resources Effurun, Nigeria.

(e-mail: jokhaifoh@yahoo.com) 
of the operating points in flow, pressure, and temperature unit without giving account for change in the composition of gas.

[4] worked on the effect of anti-surge valve and bypass flow control mechanism on the performance of Turbocharged spark ignition engine at altitude using the computer aided design simulation tool for estimating the performance of the system. The researchers made use of GT-POWER software tool for modelling the performance of spark ignition internal combustion engines and their results showed that engine power is expected to drop at high altitude. Furthermore, as they tend to avoid surge from occurring in the process of turbocharging, a mechanism known as anti-surge valve mechanism in the compressor has been determined to control the amount of fuel coming into the engine while the antisurge control mechanism controls the extra flow rate and bypasses the extra flow rate of the compressor. The limitation of this work was in the simulation result as an error occurred for both power and fuel use.

Since gas compression package is an important system in process of distributing gas. The PERTAMINA EP which is a state-owned company managing the oil and gas upstream took up a project of purchasing and installing compressor facilities in which [5] did a dynamic analysis to observe the performance of compressor for ensuring safety during use and avoid the phenomenon of surge as this may damage the components on the inside of the centrifugal compressor if not prevented. Hence, an anti-surge controller which manipulates anti surge valve to provide actions in the recycle lines when surge happens was considered and a PID controller was also considered for controlling and stabilizing the system. They ran their simulations with several scenarios based on likely issues that may occur in a gas compression system after the dynamic model of the plant have been developed. The configuration of their gas compression system was designed and modified to avoid surge phenomenon and compensate disturbances.

[6] presented fundamentals such as surge detection, dynamic centrifugal compressor, and surge cycle. They went further to describe an anti-surge and performance controllers with the importance of the control strategy, anti-surge control response modes. They also considered recycle piping and valve sizing in their work with the requirement of hot gas bypass valve requirement being discussed also.

Hence, the goal of this work is to design and implement a modified anti-surge control valve system with high sensitivity that can respond to control variables within three seconds of detection for a Mixed Refrigerant compressor. This research is also centered on the previous research work shown above but with better modifications.

\section{RESEARCH METHODOLOGY}

This research employed software tools like the Intergraph Smart Instrumentation (SPI INtools) software for drawing the schematic diagram, Lucidchart for flow diagram and excel for collating historical and test data.

The investigation of the ASCV was carried out at an Industrial Plant Complex in Bonny Island situated at Rivers State of Nigeria with coordinates: $4.4239^{\circ} \mathrm{N}, 7.2437^{\circ} \mathrm{E}$. The measurements, design and implementation were performed around the compressor deck of Train One Mixed Refrigerant compressor in the plant. Fig. 1 shows the measurement environment dimensioned $1.8 \mathrm{~km}$ by $1.3 \mathrm{~km}$ an area of $2.27 \mathrm{~km}^{2}$

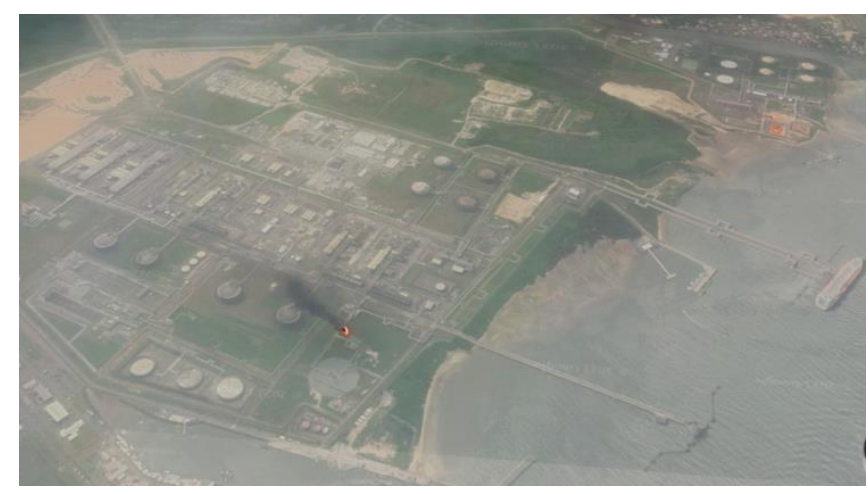

Fig. 1. Area of study

The equipment Size $-300 \mathrm{sq}$ inch

specification and Volume -28 gallons i.e., 2310 cubic inch schematic

Stroke - 24 inches

diagram (Fig. 2) Diameter -20 inches

for the ASCV are Spring-none

as Air action - close

follows:Actuator: Stroke time -10 s for control 1s for trip

Valve: $\quad$ Size $-30 \times 42$ inch

Type - angle valve

Rating - 300\#

Series of data of the performance response time of the ASCV system were collated for the following scenarios:

(a) Initial setup: This involves the collation of data over a four-year period from 2015 to 2019 due to the failure of the anti-surge valve.

(b) Modified set-up: This involves data collection after modification of the system for a period of one-year between 2020 and 2021. This is required for analysis and subsequent justification for the modification of the ASCV.

\section{DESIGN OF ModIFIED ASCV}

In carrying out this design, consideration was done independently for the three modes of operation of the ASCV ensuring that the three conditions and outcome were achieved irrespective. The specification of the ASCV itself is not altered as the control valve and actuator remain the same. However, the actuation control was improved on by remodeling it. Fig. 3 shows a schematic design of the modified anti-surge control valve.

The three design modes of operation for consideration include:

1. Mode 1: SOV trip (De-energized) - Valve fails to fully OPEN position.

2. Mode 2: Loss of Control Air Supply - Valve fails to fully Open position.

3. Mode 3: Normal Valve Control - Valve available for modulating service as required by controller output.

The control algorithm of the designed ASCV is represented by the flow chart shown in Fig. 4. 


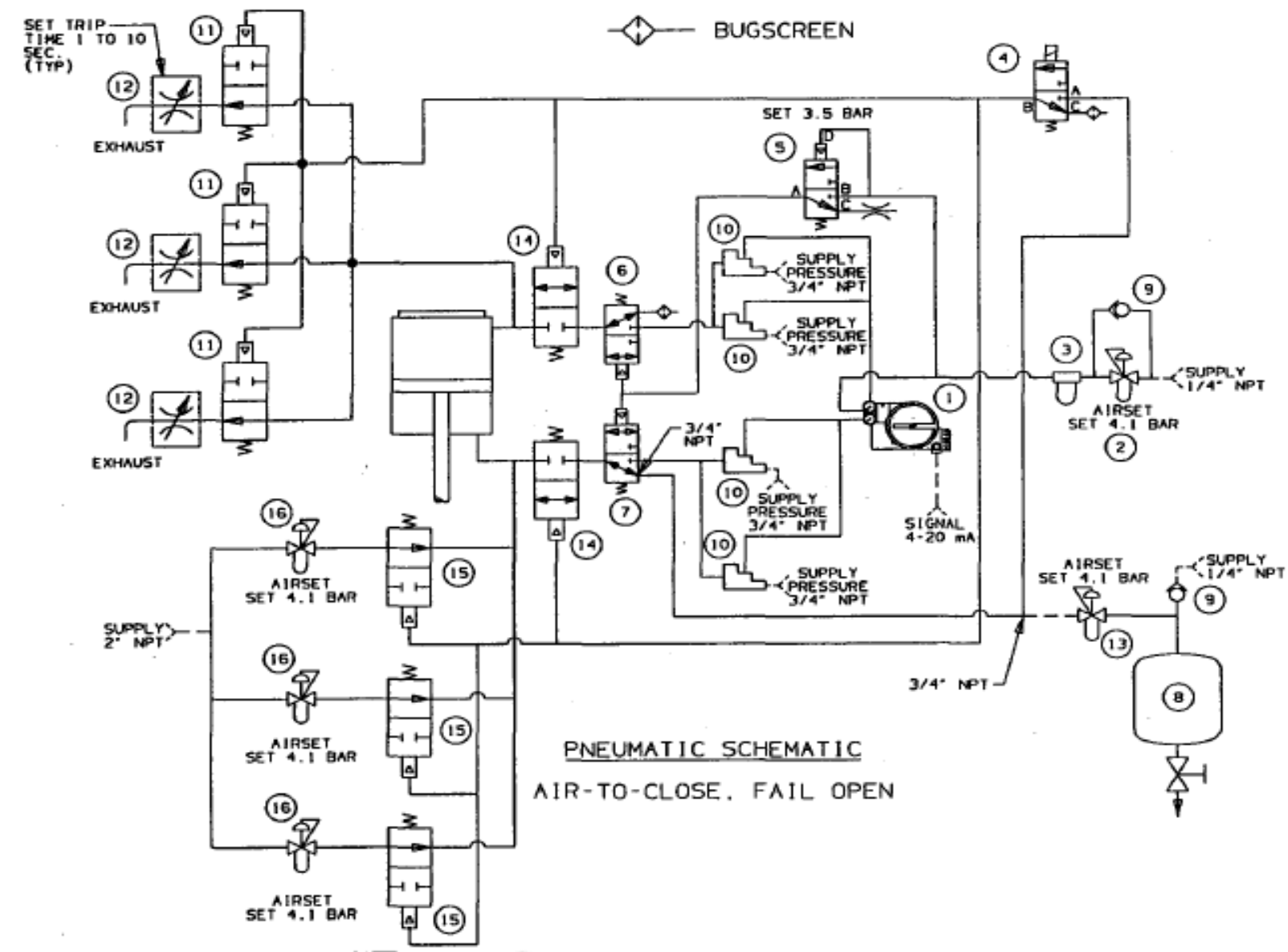

Fig. 2. Design of the initial anti-surge system.

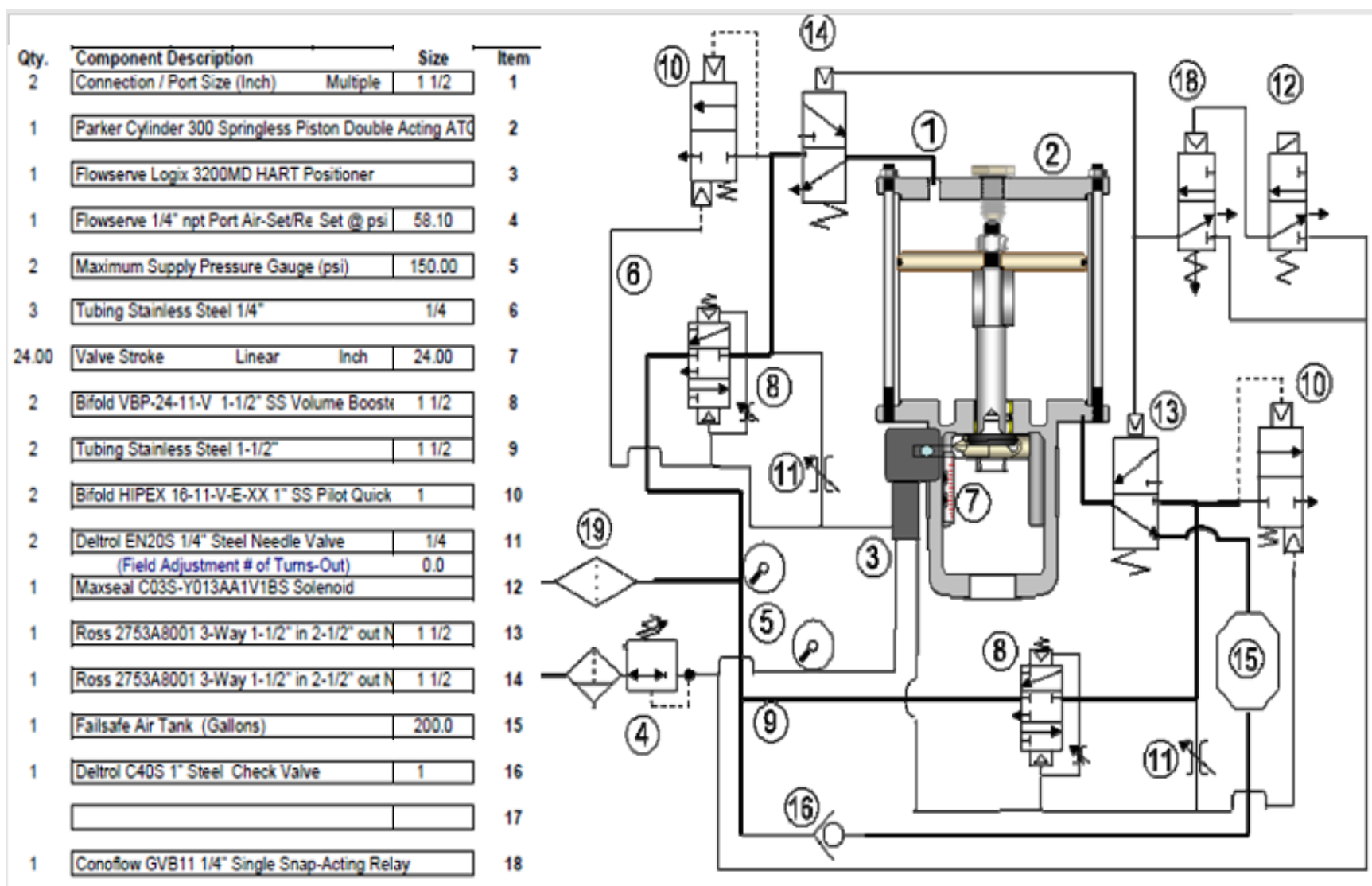

1 Norgren 5um 2" Port F47-C21-A0DA Coalesd 2 19 19

Fig. 3 Schematic diagram of modified ASCV design. 


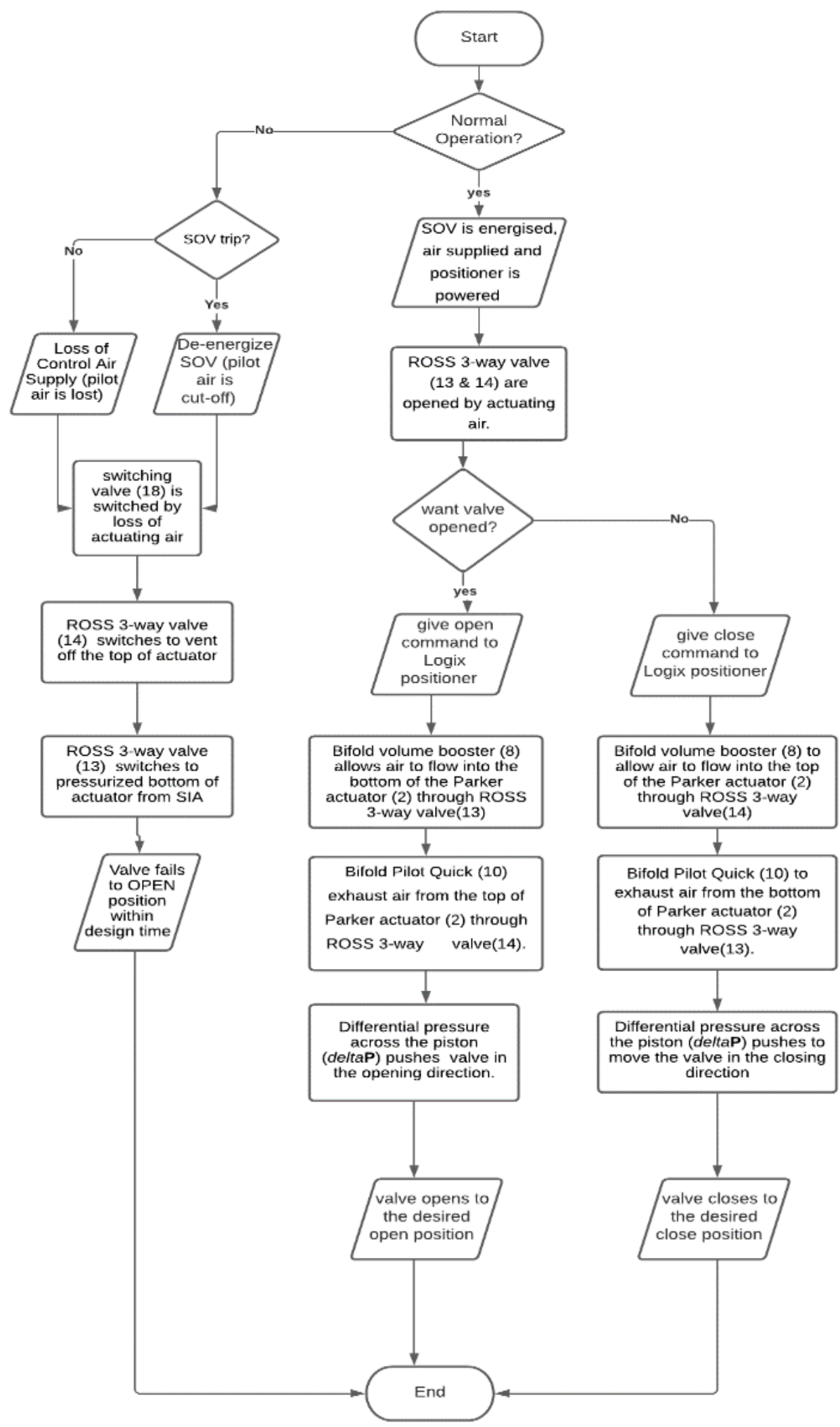

Fig. 4. Flow diagram showing design mode of operation of ASCV.

The novel components employed for this work to achieve an improved response time are explained below:

1) Bifold Pilot Quick Exhaust Labelled (10)

The schematic diagram of the Bifold Pilot Quick Exhaust is shown in Fig. 5. Flow through the Bifold Pilot Quick Exhaust (10) is proportional to the differential pressure actuating it. Summary of its operation is thus:
- when $\mathrm{P}>=\mathrm{AC}$ : item 10 is closed. Therefore, Actuator NOT exhausted

- when $\mathrm{P}<\mathrm{AC}$ : item 10 is open. Therefore, Actuator exhausted. This condition is also called Pressure Relief $(\mathrm{AC}>\mathrm{P})$ where item 10 Opens to exhausts excess pressure in actuator until $\mathrm{AC}=\mathrm{P}$. 


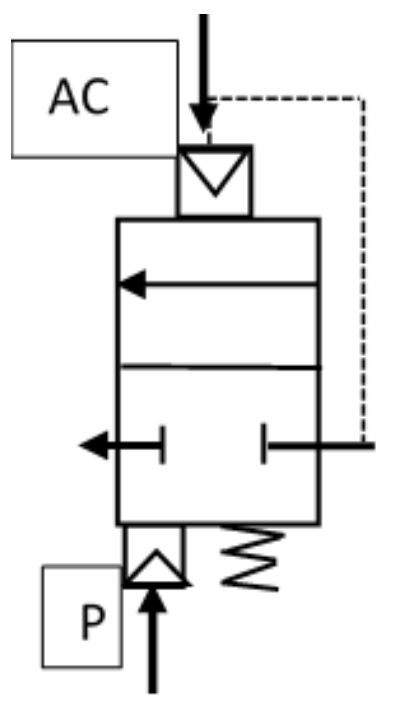

Fig. 5. Bifold pilot quick exhaust.

where $\mathrm{P}=$ positioner port pressure and $\mathrm{Ac}=$ actuator pressure.

\section{2) Bifold VBP-24-11-V; Volume Booster Labelled (8)}

The Bifold Volume Booster converts a low volume pressure signal into a 1:1 ratio high volume output. It is specifically designed for both modulating and "on/off" pilot pressure signals.

Fig. 6 shows the schematic of this volume booster.

$\mathrm{P}=$ Pilot pressure (2-10bar range), 1=Supply pressure, 2=Output pressure

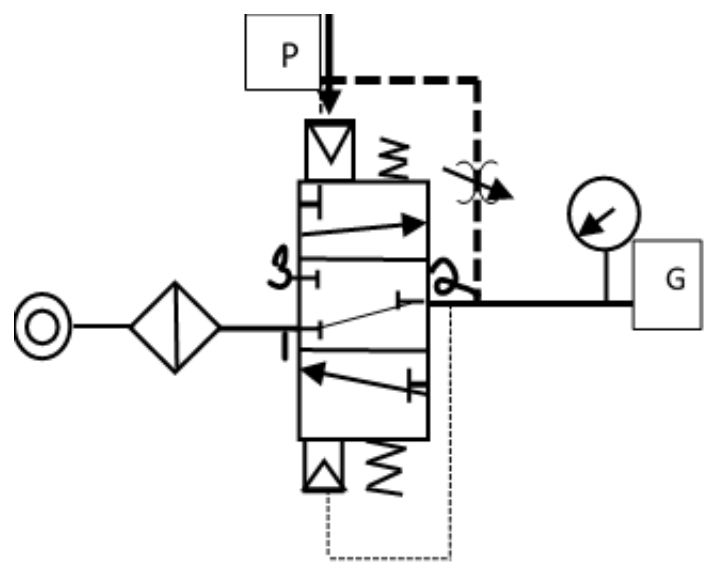

Fig. 6. Bifold volume booster.

a. When a low volume pilot pressure signal of 2 to $10 \mathrm{barg}$ is applied to the sensing port $\mathrm{P}$, the main valve assembly opens to allow high volume flow from the main inlet port 1 to the outlet port 2. This is illustrated in Fig. 7.

b. When the sensing assembly detects that the outlet pressure is equal to the pilot pressure, the main valve moves to the 'all ports blocked' rest position and will remain in this position until there is a change in the pilot pressure or outlet pressure. This is illustrated in Fig. 7.

c. If the sensing head detects that the outlet is higher than the pilot pressure, the high flow exhaust opens to vent the excess pressure. Fig. 8 shows this.

d. If the sensing head detects that the outlet pressure is too low, the main valve opens to recharge the system to the correct 1:1 ratio pressure.

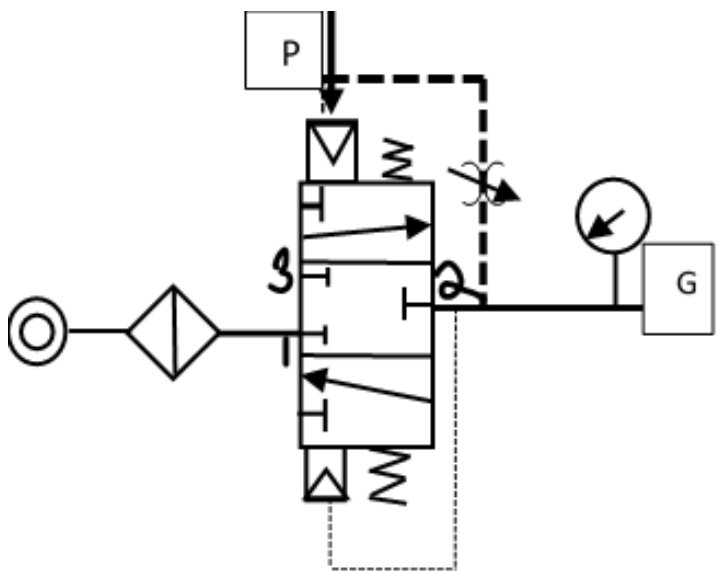

Fig. 7. Bifold volume booster scenario "b".

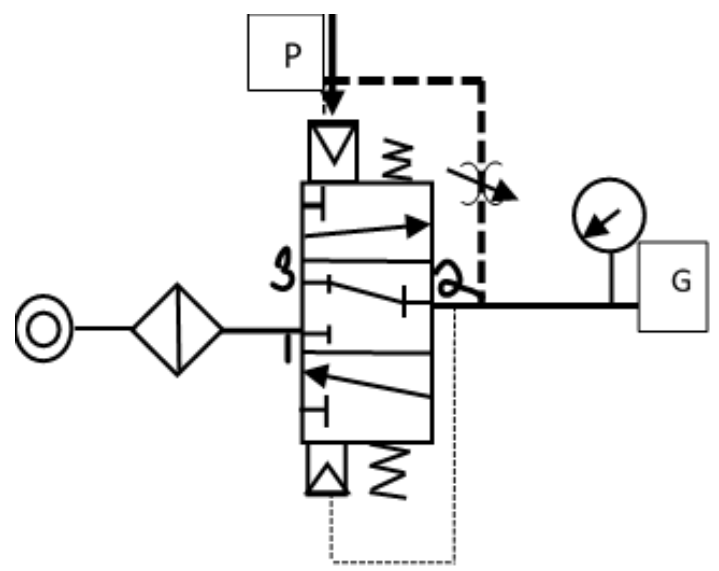

Fig. 8. Bifold volume booster scenario "c" and "d".

\section{DATA PRESENTATION AND ANALYSIS}

The response time performance for the initial set-up and modified ASCV (new design) were collated, the mean values are presented in table I. Also, Fig. 9 shows a graphical representation of the mean stroke time of both systems.

TABLE I: MEAN STROKE TIME FOR INITIAL AND MODIFIED SET-UP

\begin{tabular}{cccc}
\hline \hline Command \% & $\begin{array}{c}\text { Mean stroke } \\
\text { time for initial } \\
\text { setup (s) }\end{array}$ & $\begin{array}{c}\text { Mean stroke time } \\
\text { for modified setup } \\
(\mathrm{s})\end{array}$ & \% improvement \\
\hline $0-5$ & 3.76 & 0.77 & 79.5 \\
$0-10$ & 7.26 & 0.33 & 95.4 \\
$0-25$ & 7.51 & 0.34 & 95.4 \\
$25-50$ & 6.43 & 0.55 & 91.4 \\
$50-75$ & 6.77 & 0.67 & 90.1 \\
$75-100$ & 4.65 & 0.55 & 88.1 \\
$0-100$ & 9.35 & 2.21 & 76.3 \\
$100-0$ & 8.49 & 2.38 & 71.9 \\
Average percentage Improvement in Stroke Time & $\mathbf{8 6 . 0}$ \\
\hline \hline
\end{tabular}

The performance of the modified anti-surge control valve system was monitored with a software called Train tools Engineering and Operator Interface Software also known as CCC. With this software the performance of the modified setup is seen with timing precision. On the other hand, there is a less accurate software just for monitoring the ASCV movement only. This software is known as the PI Processbook simply known as PI. However, this software is not for the purpose of performance analysis but rather just for indication, that is to tell if the valve has moved or not. The movement of the ASCV can be observed on PI without timing 
accuracy because of the lag that exists on the software being a third-party software whose input is tapped from the DCS.

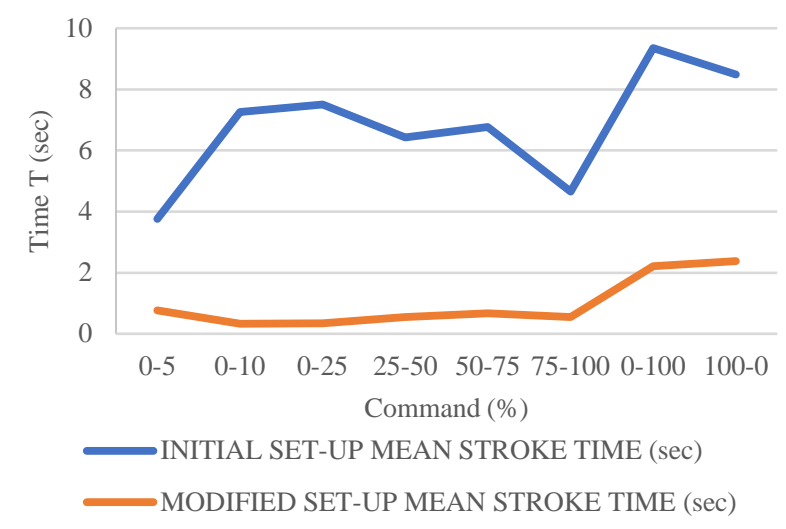

Fig. 9. Initial vs modified set-up mean stroke time.

Fig. 10 shows the user interface of the PI software and how the ASCV movement followed the issued command although without timing accuracy due to the signal lag. Fig. 12 on the other hand shows the CCC user interface and how the modified set-up responds to issued command with precision timing for performance analysis. The controller signal (command) is blue trend, while the ASCV position/response is the green trend.

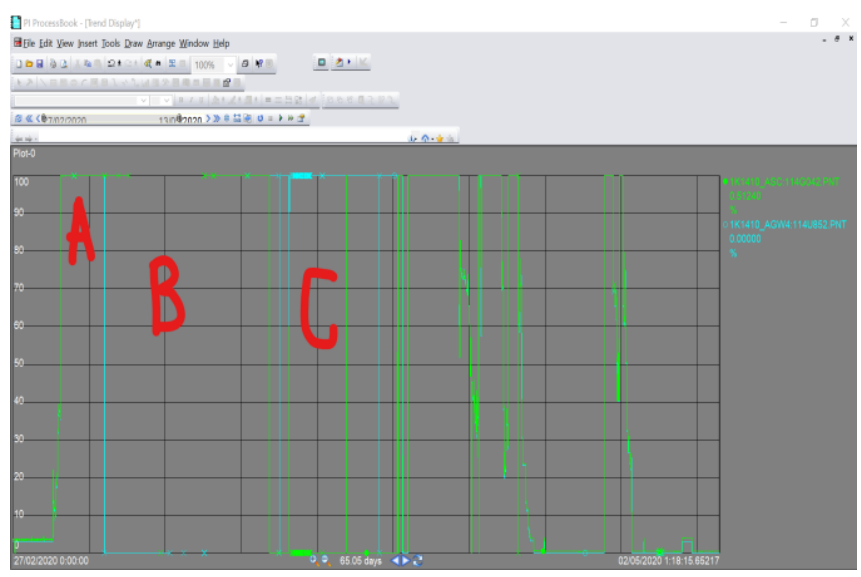

Fig. 10. PI Processbook tracking of ASCV system.

As seen from Fig. 10, at the top right corner the blue graph represents the controller command to the ASCV (114U852) while the green represents the ASCV movement itself or better put valve position (114G042). The trend has been labelled into 3 parts A, B and C. "A" represents the trend before the modification was done " $\mathrm{B}$ " represents the duration when the old system was decommissioned and hence out of service before the new system was installed, the trend can be seen to be discontinued at this point. The third part " $C$ " is the new system installed and working fine with both the controller command and the ASCV response visible. Fig. 11 zooms into a particular point to see the response of the ASCV to a given command.

As shown in Fig. 11 the controller gave a command to open from $0 \%$ to $100 \%$ (blue line) and the ASCV responded and moved $100 \%$ position (green line) after a given time.

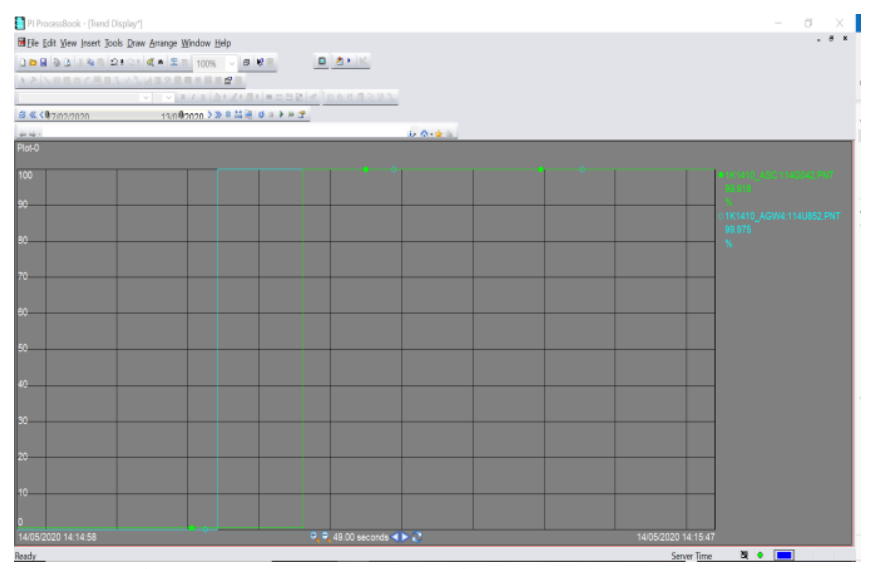

Fig. 11 ASCV response to a given controller command.

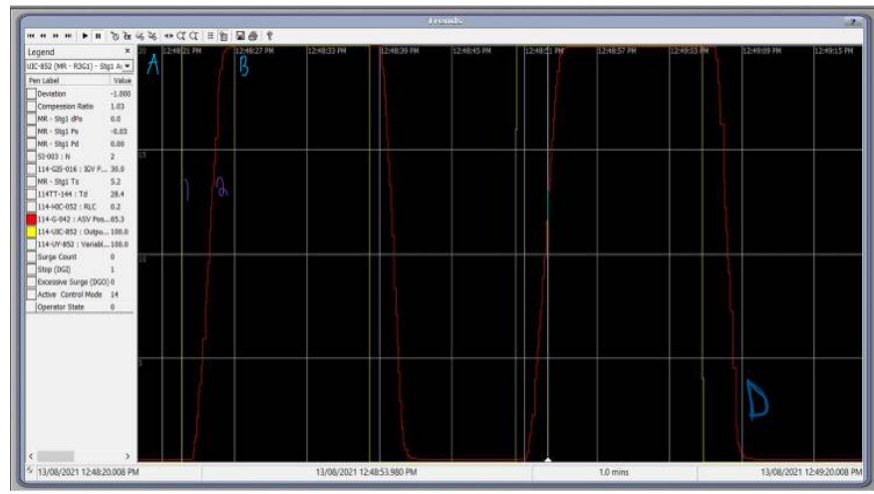

Fig. 12. CCC tracking the ASCV movement against the controller command.

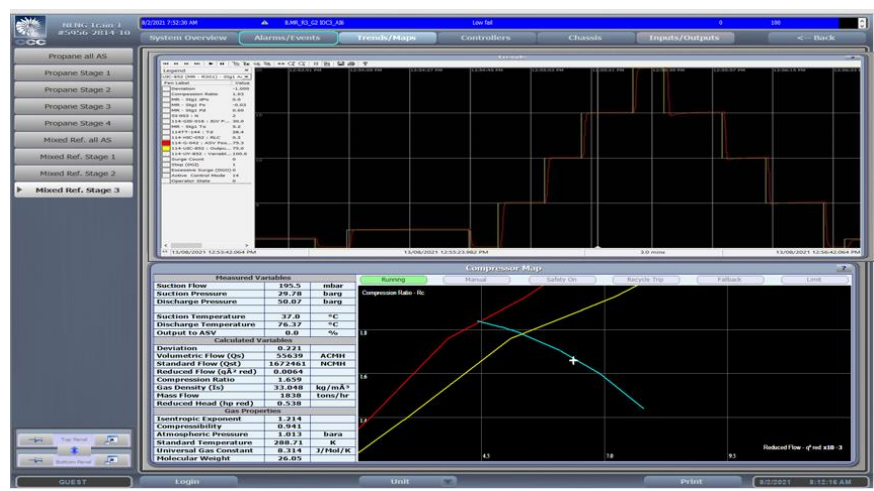

Fig. 13. Response of the ASCV to Modulated signal.

Fig. 12 shows the CCC user interface, the yellow trend is the given command (UIC-852) while the red is the valve position (G-042), this can be seen in the pen label on the lefthand side.

Fig. 13 shows another screen shot of the complete user interface.

\section{DISCUSSION AND FINDING}

From Table I it is very evident that a great improvement has been recorded in terms of the performance response time of the ASCV. The mean stroke time of the modified set-up shows improvement from the initial set-up. The table shows that the average percentage improvement from the initial setup is $86 \%$ which is a great feat. Away from the percentage improvement, the table also shows that the full range travel of the valve is now under 3 seconds as compared to the initial set-up which averages at 9 seconds. 
Fig. 9 has further been used to emphasize this improvement. A very visible difference can be spotted, the blue curve shows the stroke time recorded with the initial setup before the modification, it shows the whole range of travel from $0 \%$ to $100 \%$ of the command given. The orange-colored curve shows the recorded time for the modified system over the same travel range

Fig. 12 shows the ASCV response to command during a stroke test. The yellow line is the given command, and the red line is the valve's response. Looking at the left-hand side of the trend on Fig. 12, a command of $100 \%$ was given to the valve (the straight yellow line), and it took a certain time for the valve to attain the desired position (red line). The time difference between the yellow and red line attaining peak value is the response time and can be deducted as thus:

Time stamps of first box: $\quad \mathrm{A}=12: 48: 21$

$$
\mathrm{B}=12: 48: 27
$$

Therefore, Time interval between each box $=6$ seconds

Looking at both trends (1) and (2) (as labelled), it can be deduced, looking closely at the labelled parts that the time lag between them is less than 3 seconds.

\section{CONCLUSION}

In this paper, improving the response performance of ASCV has been the crux of this work. The use of the Bifold volume boosters and Bifold pilot quick exhaust has greatly improved the response time of the anti-surge control valve. The speed of reaction and travel time are vastly improved. For valves like the anti-surge valves that are required to respond speedily to surge detection it is soothing to know that this can be achieved with a modified design making use of the new Bifold volume boosters.

\section{ACKNOWLEDGMENT}

The authors wish to acknowledge and appreciate the staff and management of Nigeria Liquified Natural Gas Limited for their support during this work.

\section{REFERENCES}

[1] M. P. Boyce, Gas Turbine Engineering Handbook, $4^{\text {th }}$ ed. Waltham MA: Elsevier, 2012, pp. 283-290.

[2] G. Ali and G. Ghazanfarihashemi. Protecting a centrifugal compressor from surge, Pipeline \& Gas Journal, vol. 239, no. 3, March 2012.

[3] S. Mirsky, J. Mcwhirter, W. Jacobson, M. Zaghloul and D. Tiscornia Development and design of anti-surge and performance control systems for centrifugal compressors, Proceedings of the Forty-Second Turbomachinery Symposium, pp. 1-34, Texas, 2012.

[4] M. M. Doustdar, A. Goudarzi and M. G. Sooteh. Effect of anti-surge valve and bypass flow control mechanism on the Performance of turbocharged spark ignition engine at altitude, Indian journal of scientific research, 2014, vol. 1, pp. 380-388.

[5] M. L. Hakim, B. Achmad and J. P. Sutikno. (June 2018). Anti-Surge Control of Centrifugal Compressor at PT. Pertamina EP Asset 2 Field Pendopo. ASTECHNOVA 2016 International Energy Conference. [Online]. E3S Web of Conferences 42(01010). Available: http://doi.org/10.1051/e3sconf/20184201010.

[6] N. Ghaisas and T. Reitsma. (July 2016). Surge Detection and Surge. Control Systems for Centrifugal Compressors -- Part 1. Royal Belgian Institute of Marine Engineers. [Online]. Available: http://dieselpub.com/compressortech2

[7] T. L. Kumar and A. Swati. (March 2016). Anti-surge control software using general purpose programmable single loop controller. Research
Journal of Engineering Sciences. [Online]. 5(3). pp. 49-52. Available: http://www.isca.in.

[8] A. Kuzmichev. (June 2012). Compressor surge prevention control implemented on Yokogawa Stardom. The Next Generation of Control Technology. [Online]. SPC/STSC ON YOKOGAWA

STARDOM E.001.120607. Available: http:// www.ccsia.com

[9] A. Continovis, D. Pareschi, M. Mercangoez and T. Basselmann. Model predictive anti-surge control of centrifugal compressors with variablespeed drives, Proceedings of the 2012 IFAC Workshop on Automatic Control in Offshore Oil and Gas Production, Norwegian University of Science and Technology, Norway, 2012.

[10] A. A. Amin and K. Mahmood-Ul-Hasan. Advanced anti-surge control algorithm for turbine driven centrifugal compressors, Department of Electrical Engineering, University of Engineering and Technology, Lahore, 2015. 27(3). pp. 1845-11854. 